2. Jean Geffroy, Contribution d la theorie des valeurs extrêmes, Publ. Inst. Statist. Univ. Paris 7 (1958), 36-123; 8 (1959) 3-52.

3. - Localisation asymptotique du polyedre d'appui d'un echatillion Laplaçian d $k$ dimensions, Publ. Inst. Statist. Univ. Paris 10 (1961), 213-228.

4. B. V. Gnedenko, Sur la distribution limite du terme maximum d'une série aléatoire, Ann. of Math. 44 (1943), 423-453.

5. Ulf Grenander, Probabilities on algebraic structures, Wiley, New York, 1963.

6. A. Renyi and R. Sulanke, Über die konvexe Hille von $n$ züfallig gewahlten. Punkten I and II, Z. Wahrscheinkeitstheorie und Verw. Gebiete 2 (1963), 75-84; 3 (1964), 138-148.

7. A. V. Skorohod, Limit theorems for stochastic processes, Theor. Probability Appl. 1 (1956), 261-290. (Engl. transl.)

8. V. Strassen, $A n$ invariance principle for the law of the iterated logarithm, Z. Wahrscheinkeitstheorie und Verw. Gebiete 3 (1964), 211-226.

Dartmouth College

\title{
A RELATION BETWEEN A THEOREM OF BOHR AND SIDON SETS
}

BY DANIEL RIDER ${ }^{1}$

Communicated by W. Rudin, December 17, 1965

1. Introduction. In 1913, Bohr [1] proved the following theorem for Dirichlet series: if

$$
f(\sigma+i t)=\sum_{n=1}^{\infty} c(n) n^{-\sigma-i t}
$$

and if $|f(\sigma+i t)| \leqq 1$ for all $\sigma>0$, then

$$
\sum_{p}|c(p)| \leqq 1,
$$

the sum in (2) extending over all primes.

A set of positive integers $E$ will be called a Bohr set if there is a finite constant $B$ such that for every function $f$ as in (1)

$$
\sum_{n \in E}|c(n)| \leqq B
$$

1 This research was supported in part by Air Force Office of Scientific Research Grant AF-AROSR 335-63. 
It is easily seen that $E$ is a Bohr set if and only if for every finite $\operatorname{sum} f(t)=\sum c(n) n^{-i t}$

$$
\sum_{n \in E}|c(n)| \leqq B \sup _{-\infty<t<\infty}|f(t)|
$$

Let $G$ be a compact Abelian group and $E$ a subset of its dual group $\Gamma$. An E-polynomial is a trigonometric polynomial, $F$, such that $\hat{F}(\gamma)=0$ for $\gamma \notin E$ where

$$
\hat{F}(\gamma)=\int_{G} F(x) \gamma(-x) d x, \quad \gamma \in \Gamma .
$$

Here $d x$ is normalized Haar measure on $G . E$ is called a Sidon set if there is a finite constant $B$ such that

$$
\sum_{\gamma \in E}|\hat{F}(\gamma)| \leqq B \sup _{x \in G}|F(x)|=B\|F\|^{\infty}
$$

for every $E$-polynomial $F$.

Let $T^{\omega}$ be the direct product of a countably infinite collection of circles. $T^{\omega}$ is a compact Abelian group with dual group $Z^{\omega}$. Each $\gamma \in Z^{\omega}$ is given by a sequence of integers $\left\{\alpha_{k}\right\}$ where only a finite number of the $\alpha_{k}$ are not zero. $M\left(T^{\omega}\right)$ is the space of regular Borel measures, $\mu$, on $T^{\omega}$ with finite total variation $\|\mu\| . \hat{\mu}$ is the FourierStieltjes transform of $\mu$.

In this note we give a characterization of Bohr sets in terms of Sidon sets in $Z^{\omega}$ and certain measures on $T^{\omega}$. It is then possible to obtain a sufficient arithmetic condition for Bohr sets.

2. The relation between Bohr sets and Sidon sets. $P$ will denote the positive cone of $Z^{\omega}$. Let $p_{1}, p_{2}, \cdots$ be the primes. If $n$ is an integer and $n=\prod p_{j}^{\alpha_{j}}$, then we associate $n$ with the element $\gamma_{n}=\left(\alpha_{1}, \alpha_{2}, \cdots\right)$ of $P$. For a set of positive integers $E, \hat{E}=\left\{\gamma_{n}: n \in E\right\}$.

To a function $f(t)=\sum c(n) n^{-i t}$ we associate the function $F(x)$ $=\sum c(n) \gamma_{n}(x)$ on $T^{\omega}$. Bohr noticed the following: if $\phi:(-\infty, \infty) \rightarrow T^{\omega}$ by

$$
\phi(t)=\left(\exp \left(-i t \log p_{1}\right), \exp \left(-i t \log p_{2}\right), \cdots\right)
$$

then $\gamma_{n}(\phi(t))=n^{-i t}$ so that $F(\phi(t))=f(t)$. Now since $\left\{\log p_{j}\right\}$ is linearly independent over the integers, $\phi(-\infty, \infty)$ is dense in $T^{\omega}$. Thus

$$
\|F\|_{\infty}=\sup _{-\infty<t<\infty}|f(t)|
$$


TheOREM. $A$ set of positive integers $E$ is a Bohr set if and only if

(a) $\hat{E}$ is a Sidon set in $T^{\omega}$, and

(b) there is a measure $\mu \in M\left(T^{\omega}\right)$ such that

$$
\begin{aligned}
\hat{\mu}(\gamma)=1 & \text { if } \gamma \in \hat{E}, \\
=0 & \text { if } \gamma \in P-\hat{E} .
\end{aligned}
$$

Proof. Let $F(x)=\sum \hat{F}\left(\gamma_{n}\right) \gamma_{n}(x)$ be a $P$-polynomial and let $f(t)=\sum \hat{F}\left(\gamma_{n}\right) n^{-i t}$.

If $E$ is a Bohr set then by (7)

(9) $\sum_{\gamma \in \hat{E}}|\hat{F}(\gamma)|=\sum_{n \in E}\left|\hat{F}\left(\gamma_{n}\right)\right| \leqq B \sup _{-\infty<t<\infty}|f(t)|=B\|F\|_{\infty}$.

Thus if $b$ is a function on $\hat{E}$ and $|b(\gamma)| \leqq 1$ then $L(F)$ $=\sum_{\gamma \in \hat{E}} b(\gamma) \hat{F}(\gamma)$ is a bounded linear functional on the $P$-polynomials with norm at most $B$. By the Hahn-Banach and Riesz representation theorems there is a measure $\mu \in M\left(T^{\omega}\right)$ with

$$
\begin{aligned}
\hat{\mu}(\gamma) & =b(\gamma), & & \gamma \in \hat{E}, \\
& =0, & & \gamma \in P-\hat{E} .
\end{aligned}
$$

By [3, Theorem 5.7.3], $\hat{E}$ is a Sidon set; by taking $b \equiv 1$ we obtain the measure for (b).

Conversely suppose (a) and (b) are true for $E$ and let $f(t)$ $=\sum c(n) n^{-i t}$ be a finite sum. By (a) and the proof of $[3$, Theorem 5.7.3] there is $\nu \in M\left(T^{\omega}\right)$ with $\|\nu\| \leqq B$ (B depends only on $E$ ) and $c(n) \hat{\nu}\left(\gamma_{n}\right)=|c(n)|$ for $n \in E$. Let $\mu$ be as in (b) and $*$ denote ordinary convolution. Then

$$
\begin{aligned}
\sum_{n \in E}|c(n)| & =\mu * \nu * \sum c(n) \gamma_{n}(0) \\
& \leqq B\|\mu\|\|\mid\| F \|_{\infty} \\
& \leqq B^{\prime} \sup _{-\infty<t<\infty}|f(t)| .
\end{aligned}
$$

Corollary. Let $E=\left\{n_{1}, n_{2}, \cdots\right\}$ be a set of positive integers satisfying

(c) $\left\{\log n_{j}\right\}$ are linearly independent over the integers, and

(d) if $n$ is a positive integer, $\left\{\beta_{j}\right\}$ is a collection of integers, $\sum \beta_{j}=1$, and $n=\prod n^{\beta_{i}}$ then $n \in E$.

Then $E$ is a Bohr set.

PRoof. It follows from (c) that if $k_{1}<k_{2}<\cdots<k_{s}$ then $0 \neq \pm \gamma_{n_{k_{1}}}$ $\pm \gamma_{n_{2}} \pm \cdots \pm \gamma_{n_{k_{e}}}$. Thus by [2, Theorem 1.5], $\hat{E}$ is a Sidon set. 
Let $H=\left\{\gamma \in Z^{\omega}: \gamma=\sum \beta_{j} \gamma_{n_{j}}, \beta_{j}\right.$ integers, $\left.\sum \beta_{j}=1\right\} . H$ is a coset of a subgroup of $Z^{\omega}$ and by (d) $\hat{E}=H^{\prime} \cap P$. By $\left[3\right.$, p. 60] there is $\mu \in M\left(T^{\omega}\right)$ such that $\hat{\mu}$ is the characteristic function of $H^{\prime} . \mu$ satisfies condition (b) of the theorem.

3. Examples. The corollary shows that there are Bohr sets which are not the finite union of sets with pairwise relatively prime elements. For example, $p_{1} p_{2}, p_{1} p_{3}, p_{4} p_{5}, p_{4} p_{6}, p_{4} p_{7}, p_{8} p_{9}, \cdots$. It is known $[3$, p. 126] that every infinite subset of a discrete group contains an infinite Sidon subset. However this is not true of Bohr sets.

EXAMPLE. Let $F=\left\{n_{j}=\left(p_{1} p_{2} \cdots p_{j}\right)^{i}\right\}$. Then $F$ contains no infinite Bohr subset.

In fact $F$ contains no infinite subset for which there is a measure satisfying (8). For suppose $E=\left\{n_{j_{1}}, n_{j_{2}}, \cdots\right\}$ and $\hat{\mu}$ satisfies (8). Let $\mu_{k}$ be the translation of $\mu$ such that

$$
\hat{\mu}_{k}(\gamma)=\hat{\mu}\left(\gamma+\gamma_{n_{j_{k}}}\right) .
$$

$\left\{\mu_{k}\right\}$ has a weak star convergent subsequence to a measure $\nu \in M\left(T^{\omega}\right)$ which by a lemma of Helson [3, p. 66] must be singular with respect to Haar measure.

But this is impossible since it is easily seen that

$$
\begin{aligned}
\hat{\nu}(\gamma)=1 & \text { if } \gamma=0, \\
=0 & \text { if } \gamma \neq 0
\end{aligned}
$$

so that $\nu$ must be the Haar measure.

This example also shows that the corollary is false without (d).

\section{Bibliography}

1. H. Bohr, Über die Bedeutung der Potenzreihen unendlich vieler Variabeln in der Theorie der Dirichletschen Reihen, Nachr. Akad. Wiss. Göttingen Math.-Phys. Kl. (1913), 441-448.

2. D. Rider, Gap series on groups and spheres, Canad. J. Math. (to appear).

3. W. Rudin, Fourier analysis on groups, Interscience, New York, 1962.

UNIVERSITY OF WISCONSIN AND

Massachusetts Institute of TechNology 